\title{
Survival Following Road Traffic Accidents in a Level-I Trauma Center, Parametric Versus Semi-Parametric Survival Models
}

\author{
Mahnaz Yadollahi ${ }^{1,}{ }^{*}$, Mahmood Ali Nezam Eftekhari ${ }^{1}$, Kazem Jamali ${ }^{1}$ and Forough Pazhuheian $^{1}$ \\ ${ }^{1}$ Trauma Research Center, Shahid Rajaee Hospital, Shiraz University of Medical Sciences, Shiraz, Iran
}

* Corresponding author: Mahnaz Yadollahi, Trauma Research Center, Shahid Rajaee Hospital, Shiraz University of Medical Sciences, Shiraz, Iran. Tel: +987136254206; Fax: +987136254206; Email: yadollahim@sums.ac.ir

Received 2021 February 09; Revised 2021 February 28; Accepted 2021 April 20.

\begin{abstract}
Background: Simulation studies present an important statistical tool to investigate the performance, properties, and adequacy of statistical models in pre-specified situations. The proportional hazards model of survival analysis is one of the most important statistical models in medical studies. The present study aimed to investigate the underlying one-month parametric survival model of road traffic accident (RTA) victims in a Level 1 Trauma Center in Iran using parametric and semi-parametric survival analysis models from the viewpoint of post-crash care-provider in 2017.

Methods: This retrospective cohort study (restudy) was conducted at Level-I Trauma Center of Shiraz, Iran, from January to December 2017. The parametric survival modeling was employed to inspect the multiplicative effect of all covariates on the hazard considering the fact that certain covariates acting on survival may take a non-homogenous risk pattern that can lead to the violation of proportional hazards assumption in Cox proportional hazards. Distributions of choice were Exponential, Weibull, and Lognormal. Parameters were estimated using the Akaike Information Criterion for each of the fitted models.

Results: Survival analysis was conducted on 8,621 individuals for whom the length of stay (observation period) was between 1 and 89 days. In total, 141 death occurred during this time. The log-rank test revealed inequality of survival functions across various categories of age, injury mechanism, injured body region, injury severity score, and nosocomial infections. Although the risk level in the Cox model is almost the same as that in the results of the parametric models, the Weibull model in the multivariate analysis yields better results, according to the Akaike criterion.

Conclusion: In multivariate analysis, parametric models were more efficient than other models. Some results were similar in both parametric and semi-parametric models. In general, parametric models and among them the Weibull model was more efficient than other models.
\end{abstract}

Keywords: Parametric, Road traffic accidents, Semi-parametric, Survival models

\section{Background}

Throughout the history of mankind, whether and when an event occurs over a period of time has always been a mystery that remained unraveled despite the tremendous efforts of scientists. Mathematicians, statisticians, and engineers continue to pioneer this field through the development of powerful models. Moreover, these models incorporate a range of predictors to create a satisfactory model by integrating two dependent variables (Event over Time) (1). It was not until the 1950s when health care providers became conscious of the possible application of such models in the field of medicine, specifically regarding the matte of death as an event that usually follows road traffic accidents (RTAs). Fortunately, the importance RTAs and their burden on the health systems have been investigated enough (2). The most recent, reliable, and comprehensive information in this regard comes from the WHO project on the global status of road safety (2018) signifying the fact that the Eastern Mediterranean Region (EMR) suffers from an increasing rate of road traffic deaths $(17 / 100 \mathrm{~K}$ population in 2013 to $18 / 100 \mathrm{~K}$ population in 2016).

Meanwhile, advancements in the field of technology allow for extensive data gathering around various phenomena, giving birth to mega-databases that provide more than enough information to construct reliable survival models. National trauma registries are among the representative of these databases. However, death-occurrence following a road crash can be multifactorial and hinders the assessment of the efficacy of survival modeling. Solving this equation for $\mathrm{Y}$ (whether death occurs and when), requires a comprehensive recognition of multiple X components as causes (risk factors), the most crucial of which are law enforcement on speeding (3), vehicle safety standards, drunk driving behavior, motorcycle helmet use, seat-belts use, child restraints, and post-crash care (4).

High-income countries have developed robust preventive strategies through the development of reliable injury databases which allow for timely recognition of changes in various attributes of RTAs including quality of care and survival. However, 
according to the published literature, middle/lowincome countries which bear the highest burden of RTAs have poorly constructed injury databases (5-6). Moreover, information on each incident is scattered among various minor databases of responsible bodies (7) including emergency medical services, police departments, hospitals, and forensic medicine organizations; therefore, health care policymakers are hampered from proposing effective preventive strategies. Researchers in the field of RTA in developing countries have addressed this gap through the adoption of survival models which are mainly constructed on trillions of observations from western countries' experiences. Unquestionable bibles of modern trauma research with universal popularity (8), including Abbreviated Injury Scale (AIS), Injury Severity Score (ISS) (9) as well as Trauma Revised Injury Severity Score (TRISS) are incorporated into such survival analyses (10).

One potential limiting factor of TRISS methodology is its reliance on the AIS which is seldom calculated upon hospital admission, as it often requires primary, secondary, and tertiary patient surveys to assess all injuries completely. Therefore, TRISS is rarely used for early baseline risk assessment. In addition, since the original coefficients of the TRISS are based on the Major Trauma Outcome Study data, they represent the expected outcome from trauma patients treated in the 1980s in the United States (11). Therefore, the calculation of new coefficients from actual data would be a method of choice. This deduction is supported by a growing body of evidence suggesting that predicting survival after RTA in EMR using existing models/predictors may skew one's understanding of the truth, considering the fact that the majority of the abovementioned predictors of survival in developing countries vastly differ from those in the developed world in terms of drastic differences in explanatory nature of these predictors rather than their logical/statistical contribution to survival itself. Therefore, this study aimed to investigate the underlying one-month parametric survival model of RTA victims in a level 1 trauma center in Iran with the viewpoint of post-crash care-provider using parametric and semi-parametric survival analysis models in 2017.

\section{Methods}

This single-center cross-sectional study with an International Classification of Diseases (Tenth Revision) diagnosis code of V01-V99 (2015 update) was conducted on all the hospitalized injured victims of RTAs between January 2017 and December 2017. Therefore, this study aimed to investigate the underlying one-month parameteric survival model of RTA patients and to compare parametric and nonparametric survival models to determine the best fit for the study population.

The main source of information in this study was the administrative records of Shahid Rajaee hospital, a governmental trauma referral center in Shiraz, Iran. Information was retrieved from three hospital databases and anonymized for ethical purposes. Each record was then identified using a unique ten-digit serial number for data validation and statistical analyses.

\subsection{Study Population}

The sampling method did not concern this study since the methodology involved enumeration (census) of all hospitalized patients in the emergency department or other hospital wards of this center. The inclusion criteria for the conduction of primary descriptive analysis on RTA victims included age $\geq 15$ years and sustaining injuries in traffic-related incidents (drivers/passengers of 4-wheeled vehicles, motorized 2-3 wheelers, and pedestrians) requiring emergency department admission according to ICD10-CM external causes of injury codes. The 7th character of these codes is a letter that specifies whether the external cause is related to the initial (medical care) encounter (A). However, patients with the subsequent encounter (D), or a sequela (S) of the injury incident and those suffering from burns in road crashes along with complications of previous trauma surgeries (infection) were excluded from the study. A total number of 14,054 observations met the inclusion/exclusion criteria and underwent statistical analysis after data validation.

\subsection{Measurements and Data Collection}

Upon admission, baseline demographics including identifications, a ten-digit serial number, age, gender, admission date and time, and injury mechanism were recorded by admission unit employees and ER physicians. Following discharge from the emergency room, hospital, or death-occurrence, patient records were reviewed by trained staff members of the medical records unit, who provided detailed information regarding external causes of injuries codes, injury diagnosis codes (S00-T99 up to 8 descriptions per subject), possible surgical interventions, and outcome of hospitalization (coded using ICD-10).

\subsection{Injury Descriptions and Outcomes}

The injury mechanism was assigned to an external cause of injury code according to the ICD-10 injury mortality diagnosis matrix. All records were assessed for the maximum number of injury description codes upon initiation of data validation. In total 12,790 (97.7\%) patients had one or two injury diagnosis codes. An algorithm was used for each ICD-10-CM rubric in order to extract information related to the injured body regions and severity according to the method originally described by Baker and Barell $(9$, 
13). This approach resulted in the categorization of injured body regions and allowed for the calculation of ISS. In case patients suffered from multiple injuries, a single value was reported representing the highest injured body region with respect to AIS severities. This method is denoted as cross walking from ICD codes to AIS and ISS severity measures (mapping), validity and reliability of which has been extensively addressed in the literature. The ISS was calculated using chart abstraction for all deceased individuals, irrespective of time.

The outcome of interest was the occurrence of death following hospital admission. Hospital length of stay (LOS) was calculated with increments of one (days) through determining the difference between admission and discharge/death dates. Patients were considered to have acquired nosocomial infections in case one or more positive cultures were obtained from blood, respiratory secretions, urinary system, surgical incision site, or cerebrospinal fluid after 48 hours of admission.

\subsection{Statistical Analysis and Survival Models}

Baseline variables of interest were patients' demographic and injury characteristics, as well as infectious complications of medical care. Mortality and LOS were used in survival analysis. Continuous variables including age, ISS and, LOS were checked for normality and presented by Mean \pm SD or Median (interquartile range) where appropriate, whereas categorical variables including gender, injury mechanism, injured body regions, development of nosocomial infection, and mortality were presented by frequencies and proportions (n [\%]). Patients with minor injuries requiring less than 24 hours of hospitalization and the cases who are deceased within 24 hours of admission were not included in the survival analyses.

The Cox-proportional hazards (Cox PH) regression model (semi-parametric) was then fitted to the data in order to inspect the multiplicative effect of all independent predictors on the hazard (risk of death). The estimated Cox PH regression coefficients $\left(\beta_{1}, \beta_{2}\right.$, $\ldots, \beta_{\mathrm{n}}$ ) along with exponents (hazard ratios $95 \% \mathrm{CI}$ ) were reported and examined for the associations between each risk factor (age, gender, injury mechanism, injured body region, ISS, presence of infection) and the outcome.

\subsection{Parametric Models}

Parametric survival modeling was employed for the inspection of the multiplicative effect of all covariates on hazard due to the fact that certain covariates acting on survival may take a nonhomogenous risk pattern which leads to the violation of proportional hazards assumption in Cox- $\mathrm{PH}$. Regression coefficients for survival predictors in $\mathrm{PH}$ or AFT models were estimated using underlying parametric survival distributions that suitably fitted the data where appropriate. Distributions of choice were Exponential, Weibull, and Lognormal. Estimation of the parameters, as well as the Akaike information criterion (AIC) values, were recorded for each of the fitted models. The choice of optimal statistical distributions defining the underlying time to event was based on the inspection of linearity of log-survival vs. time for exponential distribution and inspecting the $\log (-\log )$ of survival probabilities vs. log time for Weibull distribution. The statistical analysis was conducted using STATA software (version 14).

\section{Results}

This study included 14,054 injured RTA individuals with a median age of 29 years (IQR [2243]) and a male/female ratio of 2.9. Passengers or drivers of 4-wheeled vehicles comprised the majority (52.9\%) of the injured population. RTA victims had a median (IQR) injury severity of 4 (1-8) and the most severely injured anatomic regions included extremities (57.3\%) followed by head and neck (18.1\%) according to ISS body regions. Hospital LOS was $1(0-3)$ days and 170 cases died contributing to a $1.2 \%$ crude mortality rate. All baseline variables excluding gender were significant contributors to mortality (Table 1).

Survival analysis was conducted on 8,621 cases for whom LOS was between 1 and 89 days (observation period). It is worth mentioning that in 39,543 patients, the duration of hospitalization was less than one day, which was not included in the survival analysis. The long-rank test revealed inequality of survival functions across various categories of age, injury mechanism, injured body region, ISS, and nosocomial infected individuals. Individuals with higher age and ISS as well as motorcycle riders and those with head injuries showed significantly lower survival probabilities as did those who developed hospital-acquired infections during their stay.

In a fitted Cox-PH regression model, ISS was the strongest predictor of survival followed by age and central nervous system (CNS) injury, all of which inversely affected survival probabilities. Each oneunit increase in ISS and age from the baseline corresponded to an 8 and 4 percent increase in the risk of death, respectively. The risk of death during hospitalization for individuals with CNS injury was 3.5 times that of the cases with injured extremities. The development of a nosocomial infection had a protective effect on survival, after adjusting for covariates. However, the global test of the proportional hazards revealed that hazards in various groups of patients were not proportional considering ISS and nosocomial infection and injured body regions which violated the prerequisites of the Cox regression model accordingly (Table 2). 


\begin{tabular}{|c|c|c|c|c|}
\hline \multirow{3}{*}{$\begin{array}{l}\text { Variables } \\
\text { Baseline Variables } \\
\text { Age (years) }\end{array}$} & \multicolumn{2}{|c|}{ Total } & \multicolumn{2}{|c|}{ Deceased } \\
\hline & \multicolumn{2}{|c|}{ Mean \pm SD, Median (IQR), n (column \%) } & \multicolumn{2}{|c|}{ Mean $\pm S D$, Median (IQR), n (row \%) } \\
\hline & $34 \pm 15.6$ & $29(22-43)$ & $49.9 \pm 22.7$ & $49(29-70)$ \\
\hline \multicolumn{5}{|l|}{ Gender } \\
\hline Female & $3604(25.6 \%)$ & & $34(0.94 \%)$ & \\
\hline Male & $10450(74.4 \%)$ & & $136(1.30 \%)$ & \\
\hline \multicolumn{5}{|l|}{ Injury Mechanism } \\
\hline Pedestrian & 4597 (32.7\%) & & $42(0.91 \%)$ & \\
\hline Car & $7432(52.9 \%)$ & & $73(0.98 \%)$ & \\
\hline Motorcycle & $2025(14.4 \%)$ & & $55(2.72 \%)$ & \\
\hline \multicolumn{5}{|l|}{ Injured Body Region } \\
\hline Upper/Lower extremities & $4200(29.9 \%)$ & & $14(0.33 \%)$ & \\
\hline Thorax internal organs & $1157(8.2 \%)$ & & $13(1.12 \%)$ & \\
\hline Abdominal Organs/lower back/pelvis & $3780(26.9 \%)$ & & $70(1.85 \%)$ & \\
\hline $\begin{array}{l}\text { the central nervous system including } \\
\text { the vertebral column and spinal cord }\end{array}$ & $4917(35.0 \%)$ & & $73(1.48 \%)$ & \\
\hline Injury Severity Score & $5.2 \pm 6.5$ & $4(1-8)$ & $32.6 \pm 20.5$ & $25(18-38)$ \\
\hline \multicolumn{5}{|l|}{ Nosocomial Infection } \\
\hline Negative & $13760(97.9 \%)$ & & $121(0.88 \%)$ & \\
\hline Positive & $294(2.1 \%)$ & & $49(16.67 \%)$ & \\
\hline Length of Stay (days) & $2.8 \pm 5.9$ & $1(0-3)$ & $10.6 \pm 16.4$ & $2(1-15)$ \\
\hline
\end{tabular}

Table 2. Proportional hazard of death in semi-parametric Cox PH regression model

\begin{tabular}{|c|c|c|c|c|c|c|c|}
\hline \multirow{2}{*}{ Variables } & \multicolumn{3}{|c|}{ Cox-PH Regression } & \multicolumn{4}{|c|}{ Global test of PH assumption } \\
\hline & Adjusted HR (95\% CI) & Z-score & P-value & $\rho$ & $\chi^{2}$ & df & P-value \\
\hline Age (years) & $1.04(1.03-1.05)$ & 7.65 & $<0.001$ & 0.06 & 0.8 & 1 & 0.37 \\
\hline Gender (female) & $1.75(1.07-2.73)$ & 2.28 & 0.023 & -0.10 & 1.52 & 1 & 0.21 \\
\hline \multicolumn{8}{|l|}{ Injury Mechanism } \\
\hline Pedestrian & Reference & & & & & & \\
\hline Car & $0.99(0.65-1.52)$ & -0.02 & 0.98 & 0.06 & 0.57 & 1 & 0.45 \\
\hline Motorcycle & $1.46(0.93-2.34)$ & 1.60 & 0.11 & 0.02 & 0.67 & 1 & 0.41 \\
\hline \multicolumn{8}{|l|}{ Body Region } \\
\hline Upper/Lower extremities & Reference & & & & & & \\
\hline Thorax internal organs & $2.68(1.19-6.10)$ & 2.4 & 0.19 & -0.13 & 2.53 & 1 & 0.11 \\
\hline Abdominal Organs/lower back/pelvis & $4.08(2.16-7.68)$ & 4.36 & 0.67 & -0.20 & 6.21 & 1 & 0.01 \\
\hline $\begin{array}{l}\text { Central nervous system including } \\
\text { vertebral column and spinal cord }\end{array}$ & 3.5 (1.91 6.39) & 4.08 & 0.16 & -0.23 & 8.16 & 1 & 0.004 \\
\hline Injury Severity Score & $1.08(1.08-1.09)$ & 17.16 & $<0.001$ & -0.20 & 4.79 & 1 & 0.03 \\
\hline Nosocomial Infection & $0.34(0.21-0.55)$ & -4.34 & 0.02 & 0.15 & 4.56 & 1 & 0.04 \\
\hline Akaike information criterion & \multicolumn{3}{|c|}{1229.32} & & & & \\
\hline
\end{tabular}

According to the exponential and Weibull's models, the factors affecting the risk of death included age, injury to the head, and severity of the injury; however, based on lognormal survival regression, gender (male), injury mechanism (motorcycle), and face injuries also increase the risk of death in addition to the variables expressed.
Although the risk level in the Cox model is almost the same as that in the results of the parametric models, the Weibull model in the multivariate analysis has better results according to the Akaike criterion. Therefore, the results of the fitting index of the AIC model indicate that Weibull's parametric model is more efficient than other functions (Table 3).

\begin{tabular}{|c|c|c|c|}
\hline \multirow[t]{2}{*}{ variables } & \multicolumn{3}{|c|}{ Hazard ratio $(95 \% \mathrm{CI})$} \\
\hline & Weibull & Exponential & Lognormal \\
\hline Age & $1.03(1.02-1.04)$ & $1.04(1.03-1.05)$ & $1.03(1.02-1.04)$ \\
\hline Gender (Female) & $1.55(0.97-2.49)$ & $1.59(0.99-2.55)$ & $1.44(1.11-1.65)$ \\
\hline \multicolumn{4}{|l|}{ Injury mechanism } \\
\hline Pedestrian & & Reference & \\
\hline Car & $1.07(0.7-1.64)$ & $1.08(0.71-1.65)$ & $1.05(0.59-1.37)$ \\
\hline Motorcycle & $1.47(0.92-2.35)$ & $1.50(0.94-2.38)$ & $1.39(1.03-1.62)$ \\
\hline \multicolumn{4}{|l|}{ Body regions } \\
\hline Upper/Lower extremities & & Reference & \\
\hline Abdominal Organs/lower back/pelvis & $1.66(0.82-3.36)$ & $1.63(0.81-3.30)$ & $1.45(0.94-1.72)$ \\
\hline Thorax internal organs & $0.72(0.31-1.68)$ & $0.72(0.31-1.67)$ & $0.60(0.28-1.35)$ \\
\hline Central nervous system including vertebral column and spinal cord & $2.28(1.54-3.38)$ & $2.29(1.54-3.40)$ & $1.64(1.46-1.76)$ \\
\hline Injury Severity Score & $1.01(1.00-1.02)$ & $1.01(1.00-1.02)$ & $1.01(1.00-1.02)$ \\
\hline Infection & $0.98(0.65-1.49)$ & $1.21(0.84-1.75)$ & $1.03(0.47-1.39)$ \\
\hline Akaike information criterion & 1201.52 & 1206.05 & 1223.43 \\
\hline
\end{tabular}


According to the Kaplan-Meier survival analysis, the log-rank test indicated a significant difference in the survival function between injured motor-vehicle occupants, motorcycle riders, and pedestrians involved in the crashes. The cumulative percentage of survived victims was higher for motorcycle riders than car accidents and pedestrians at any given point in time (Figure 1).

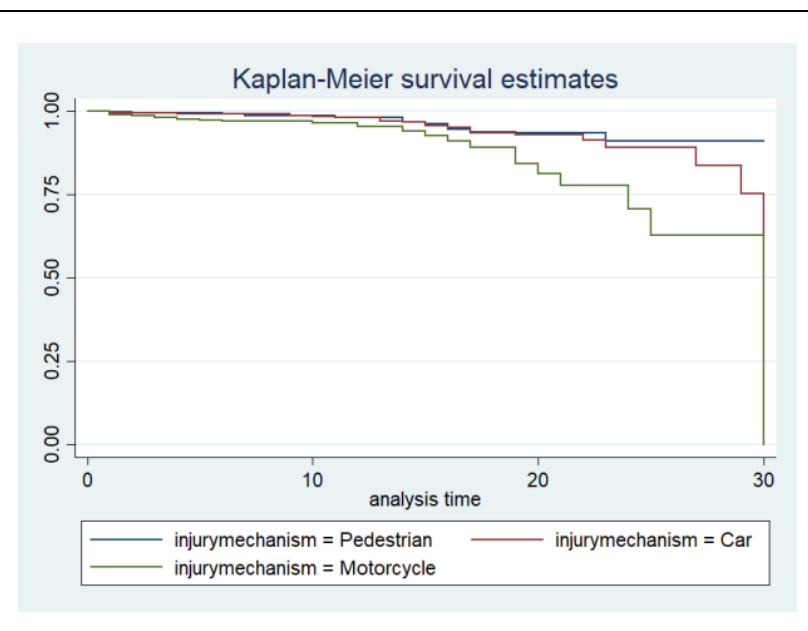

Figure 1. Survival function according to the mechanism of injury. The figure presents the time distribution of the cumulative percentage of survivors

\section{Discussion}

This study evaluated the risk ratio of death caused by road traffic injuries based on risk factors. Furthermore, parametric and semi-parametric survival functions had been applied and compared to choose the best model according to AIC criteria comparison.

Based on the obtained results, the relative risk of death significantly increases with age. Numerous trauma studies have investigated the relationship between demographic variables, especially the variable of age and mortality independent of other risk factors in trauma patients (14-15). Moreover, in line with the result of the study conducted by $\mathrm{Y}$ Chung et al. the death hazard ratio in males is higher than that in females (16). The result of the KaplanMeier plot and survival functions indicated that motorcycle and car accidents have a higher death risk ratio compared to pedestrians. This result is consistent with the result of another study conducted by Ming-Der Li indicating that cumulative proportion survival in motorcycle injuries are significantly higher than that in car accidents (17). In addition, the relative risk of death in head and neck injuries is significantly higher than that in extremities based on all parametric and semiparametric models. However, in a study performed by Seid Met l. the death ratio in individuals with head and neck injury is higher than that in other types of injuries (18). Moreover, in a study conducted by Farah A. Mansuri et al., it was revealed that head and neck injuries are more frequent injuries in any mechanism of accidents (19). The higher risk of death in head and neck injuries can be justified by the severity of the damage in these areas.

Medical researchers often evaluated the death ratio by logistic regression with odds of death, whereas, logistic regression is sensitive to the number of events. In death events, logistic regression for the sequence of rare events does not have the goodness of fit to data overlay (20-21). In a road traffic dataset with a low ratio outcome (death to alive), hazard ratio coefficients are more accurately predicted. It should be noted that the results of the Cox model will not be reliable in case the assumption of proportional hazard is not satisfied. Therefore, parametric models, such as Normal, Weibull, and Exponential can provide better choices in such situations. One of the key assumptions of the Cox model is the proportional hazards function assumption. Specifically, the model assumes that each covariate has a multiplicative effect in the hazards function that is constant over time, which is not required in parametric models. This study aimed to compare the Cox regression and parametric models in the survival analysis of patients with road traffic injuries and compare the models using the Akaike criterion. Based on the results, Weibull distribution has the best fit to data among parametric models. Another study revealed that lognormal is the best survival model to evaluate the effect of some intervention in a study, especially in a survey data. However, Weibull model was antecedent to other models in the present study. The study conducted by Askarishahi et al. showed that the Cox model was best for determining the factors that influence the diagnosis of retinopathy in type II diabetes, according to the Akaike information criterion (22). In addition, the study performed by Stephen M. DiRusso et al. compared Cox regression and artificial neural networks for the prediction of survival in pediatric trauma patients. The result showed that artificial neural network was more efficient in predicting survival compared to the Cox model and logistic regression (20).

Moreover, the study performed by Poorhosseingholi et al. compared Cox Regression and Parametric Models in Patient Survival Analysis of gastric Cancer and showed that the normal log model had the best fit among parametric models and can be used as a substitute for the Cox model in survival analysis of patients with gastric cancer (23). Moghimi-Dehkordi et al. compared Cox and parametric models and showed that although the hazard ratio in the Cox model and the parametric ones are approximately similar, the Weibull and Exponential models are the most favorable for the 
survival analysis according to Akaike Information Criterion (24). However, in the present study, parametric models had higher efficiency compared to proportional hazard assumptions.

In this study, the assumption of proportional hazards was established for all models, and the analysis of residue curves showed that the fitting of the models was appropriate in parametric models. Based on the results, parametric models were more efficient than other models in multivariate analysis. Some results were similar for both parametric and semi-parametric models; However, parametric models and among them the lognormal model were more efficient in general. Regarding the limitations of the present study, one can refer to the high percentage of right censoring. It is worth mentioning that, parametric models are more suitable for fitting when the percentage of censorship does not increase from 40 to $50 \%$ (13). In addition, the cases of deaths without ID numbers or individuals with an ID number who did not meet the predefined rules were not considered in the analysis. Furthermore, individuals involved in multiple crashes were not considered as well due to the fact that complicated injury sequelae might have occurred between crashes. Eventually, alcohol consumption that was associated with more severe crash injuries was not considered due to the unavailability of the relevant data.

\section{Conclusion}

Although the Cox parameter estimations are well known to researchers in the field of medical sciences, the results in accelerated failure times can be interpreted as a relative risk that is not unknown to medical scientists. Therefore, these parameters can be interpreted as acceleration or deceleration factors in the interpretation of Cox's hazard ratio. These parametric models can be easily implemented using maximum likelihood estimators. Moreover, they allow the researchers to explore data through the exploration of different relationships, such as linear trends, nonlinear ones, or interactions. The proportional hazard assumption does not hold these methods which leads to acceptable conclusions.

\section{Acknowledgments}

This study was extracted from the thesis authored by Dr. Eftekhari for partial fulfillment for the specialty in emergency medicine and was supervised by Dr.Yadollahi.

\section{Footnotes}

Author's contributions: M.C performed the experiments and supervision. C.D analyzed the data. J.P designed experiments and co-wrote the paper.
Conflicts of Interest: The authors have no conflict of interest to declare.

Ethical Approval: This study was approved by Yizheng People's Hospital, Jiangsu Province, China.

Funding/Support: Yizheng People's Hospital, Jiangsu Province, China.

Informed Consent: Informed consent was not necessary for this in vitro study.

\section{References}

1. Kaplan EL, Meier P. Nonparametric estimation from incomplete observations. J Am Statist Assoc. 1958;53(282): 457-81.

2. Bazargan-Hejazi S, Ahmadi A, Shirazi A, Ainy E, Djalalinia S, Fereshtehnejad SM, et al. The burden of road traffic injuries in Iran and 15 surrounding countries: 1990-2016. Arch Iran Med. 2018;21(12):556-65. [PubMed: 30634852].

3. Wilson C, Willis C, Hendrikz JK, Le Brocque R, Bellamy N. Speed cameras for the prevention of road traffic injuries and deaths. Cochrane Database Syst Rev. 2010;11:Cd004607. doi: 10.1002/14651858.CD004607.pub4. [PubMed: 21069682].

4. Goldman S, Siman-Tov M, Bahouth H, Kessel B, Klein Y, Michaelson $\mathrm{M}$, et al. The contribution of the Israeli trauma system to the survival of road traffic casualties. Traffic Inj Prev. 2015;16(4):368-73. doi: 10.1080/15389588.2014.940458. [PubMed: 25133878].

5. Chokotho LC, Matzopoulos R, Myers JE. Assessing quality of existing data sources on road traffic injuries (RTIs) and their utility in informing injury prevention in the Western Cape Province, South Africa. Traffic Inj Prev. 2013;14(3):267-73. doi: 10.1080/15389588.2012.706760. [PubMed: 23441945].

6. Karkee R, Lee AH. Epidemiology of road traffic injuries in Nepal, 2001-2013: systematic review and secondary data analysis. BMJ Open. 2016;6(4):e010757. doi: 10.1136/ bmjopen-2015-010757. [PubMed: 27084283].

7. Hu G, Baker T, Baker SP. Comparing road traffic mortality rates from police-reported data and death registration data in China. Bull World Health Organ. 2011;89(1):41-5. doi: 10.2471/BLT.10.080317. [PubMed: 21346889].

8. Lefering R. Trauma scoring systems. Curr Opin Crit Care. 2012;18(6):637-40. doi: 10.1097/MCC.0b013e3283585356. [PubMed: 22918259].

9. Baker SP, O'Neill B, Haddon W Jr, Long WB. The injury severity score: a method for describing patients with multiple injuries and evaluating emergency care. J Trauma. 1974;14(3):187-96. [PubMed: 4814394].

10. Boyd CR, Tolson MA, Copes WS. Evaluating trauma care: the TRISS method. Trauma score and the injury severity score. $J$ Trauma. 1987;27(4):370-8. [PubMed: 3106646].

11. Champion HR, Copes WS, Sacco WJ, Lawnick MM, Keast SL, Bain LW Jr, et al. The major trauma outcome study: establishing national norms for trauma care. J Trauma. 1990;30(11):1356-65. [PubMed: 2231804].

12. Yadollahi M, Paydar S, Sabetianfard Jahromi G, Khalili H, Etemadi S, Abbasi $\mathrm{H}$, et al. Types and causalities in dead patients due to traumatic injuries. Arch Trauma Res. 2015; 4(1):e26028. doi: 10.5812/atr.26028. [PubMed: 25798419].

13. Loftis KL, Price JP, Gillich PJ, Cookman KJ, Brammer AL, St Germain T, et al. Development of an expert based ICD-9-CM and ICD-10-CM map to AIS 2005 update 2008. Traffic Inj Prev. 2016;17(Suppl 1):1-5. doi: 10.1080/15389588.2016.1191069. [PubMed: 27586094].

14. Sadeghi-Bazargani H, Ayubi E, Azami-Aghdash S, Abedi L, Zemestani A, Amanati L, et al. Epidemiological patterns of road traffic crashes during the last two decades in Iran: a review of the literature from 1996 to 2014. Arch Trauma Res. 2016; 5(3):e32985. doi: 10.5812/atr.32985. [PubMed: 27800461].

15. Yadollahi M, Paydar S, Sabetianfard Jahromi G, Khalili H, Etemadi S, Abbasi $\mathrm{H}$, et al. Types and causalities in dead patients due to traumatic injuries. Arch Trauma Res. 2015; 
4(1):e26028. doi: 10.5812/atr.26028. [PubMed: 25798419].

16. Chung Y. Development of an accident duration prediction model on the Korean Freeway Systems. Accid Anal Prev. 2010;42(1):282-9. doi: 10.1016/j.aap.2009.08.005. [PubMed: 19887169].

17. Li MD, Doong JL, Huang WS, Lai CH, Jeng MC. Survival hazards of road environment factors between motor-vehicles and motorcycles. Accid Anal Prev. 2009;41(5):938-47. doi: 10.1016/j.aap.2009.05.009. [PubMed: 19664430].

18. Seid M, Azazh A, Enquselassie F, Yisma E. Injury characteristics and outcome of road traffic accident among victims at Adult Emergency Department of Tikur Anbessa specialized hospital, Addis Ababa, Ethiopia: a prospective hospital based study. BMC Emerg Med. 2015;15(1):10. doi: 10.1186/s12873-0150035-4. [PubMed: 25990560].

19. Mansuri FA, Al-Zalabani AH, Zalat MM, Qabshawi RI. Road safety and road traffic accidents in Saudi Arabia: a systematic review of existing evidence. Saudi Med J. 2015;36(4):418-24. doi: 10.15537/smj.2015.4.10003. [PubMed: 25828277].

20. DiRusso SM, Chahine AA, Sullivan T, Risucci D, Nealon P, Cuff S, et al. Development of a model for prediction of survival in pediatric trauma patients: comparison of artificial neural networks and logistic regression. J Pediatr Surg. 2002; 37(7):1098-104. doi: 10.1053/jpsu.2002.33885. [PubMed: 12077780].

21. Hachamovitch R, Hayes SW, Friedman JD, Cohen I, Berman DS. Comparison of the short-term survival benefit associated with revascularization compared with medical therapy in patients with no prior coronary artery disease undergoing stress myocardial perfusion single photon emission computed tomography. Circulation. 2003;107(23):2900-7. doi: 10.1161/ 01.CIR.0000072790.23090.41. [PubMed: 12771008].

22. Askarishahi M, Keshavarzi F, AfkhamiArdakani M, Falahzadeh $\mathrm{H}$. Using parametric and Cox models in analysis of factors influencing the diagnosis of retinopathy in type II diabetes. $J$ Mazandaran Univ Med Sci. 2014;24(113):28-35.

23. Pourhoseingholi M, Pourhoseingholi A, Vahedi M, Dehkordi BM, Safaee A, Ashtari S, et al. Alternative for the cox regression model: using parametric models to analyze the survival of cancer patients. Int J Cancer Manage. 2011;4(1):1-9.

24. Moghimi-Dehkordi B, Safaee A, Pourhoseingholi MA, Fatemi R, Tabeie Z, Zali MR. Statistical comparison of survival models for analysis of cancer data. Asian Pac J Cancer Prev. 2008; 9(3):417-20. [PubMed: 18990013]. 